\title{
Listening Visits to Mothers' Suffering with Mild to Moderate Peri-Natal Depression; Critical Issues of Clinical Supervision, Training and Health Visiting Capacity to Tackle Health Inequalities
}

\section{Paul Regan*}

School of Health, University of Central Lancashire, UK

\begin{abstract}
The importance of United Kingdom health visitors' in the detection and treatment of peri-natal depression in primary care services is well documented. Evidence based policy and clinical guidelines make recommendations for the prediction, detection and treatment of women with mild to moderate depression in the postnatal period continuing up to a year after delivery. An inter-professional cross organisational practice development project aimed to assess the provision of antenatal and peri-natal mental health care within one health visiting service in accordance with NICE guidelines (2007). This paper discusses the disparity between guidelines and health visiting practice; a lack of training in listening visits or other psycho therapeutic methods, poor access and availability of clinical supervision, commissioning reforms leading to larger health visiting caseloads suggesting the service is unable to meet NICE recommendations. Lastly, the evidence suggests the scope and practice of community nurses' within health visiting teams should be expanded.
\end{abstract}

\section{Background}

The United Kingdom (UK) Health visitor implementation plan 2011-2015; a call to action (DH, 2011) suggests improving health visitors' ability to offer timely, effective interventions to mothers', children and their families within primary care services [1]. The implementation plan aims to train 4200 more health visitors' by 2015 [1] in order to counteract the effect of commissioning reforms [2] and numbers falling to 8,017 in 2010 from 10,137 in 2004 [1]. The expansion of the purchaser/ provider funding split within the UK means commissioners of services are viewed as a powerful mechanism for local funding and decision-making [1]. Primary Care Trusts (PCT's) had to become more competitive and 'fit for purpose' by reducing costs and as a result the health visiting services capacity to tackle health inequalities were reduced [3]. The McKinsey report [4] recommended reducing the mandatory workforce ratios and increase the frequency of community nurse home visits and productivity. However, the expansion of commissioning, or outsourcing to private healthcare companies within the National Health Service has led to concerns within community services [5], despite the health visitor implementation plan's attempts to rectify the errors made [1].

\section{Introduction}

Peri-natal depression (PND), previously known as postnatal depression (termed postpartum depression in North America), is a non psychotic depression meeting standardised diagnostic criteria for a minor or major depressive disorder [6]. The signs and symptoms of peri-natal depression are identical to other depressive disorders as defined by the DSM 1V (1997) criteria. The prevalence of mothers' suffering with peri-natal depression is $10-15 \%$ [7], and often poorly reported and detected with negative effects on maternal, infant and family health [6]. The National Institute for Health and Clinical Excellence (NICE) guidelines entitled Antenatal and Postnatal Mental Health aim to promote the early prediction and treatment of PND within the UK. Once NICE guidelines are published and disseminated, local healthcare Trusts are expected to develop local protocols and action plans. A survey of all UK PCT's by NICE [6] had a $48 \%$ response rate ( $n=144$ PCT's) identifying that only $54 \%$ of PCT's had a mental health training programme for health visitors' (64\% trained). NICE concluded a reasonable estimate of $25 \%$ of PCT's had a fully developed policy implemented for antenatal and postnatal mental health. What can be done at a local level is now discussed with recommendations made from the literature at the end of this paper.

\section{Practice development}

A practice development team focussed on developing an Integrated Care Pathway (ICP) and cross organisational collaboration [8]. The purpose of the team was to review and develop inter-professional referral processes (stepped/tiered care model), a local action plan/ protocol and meet the NICE guidelines [6]. The team comprised of health visitors', a psychiatrist, a manager/ facilitator, midwives, school health nurses' and community mental health nurses'. The benefit of a practice development approach allows the systematic review by practitioners on aspects of their own practice [8]. One key issue was the lack of available data collection systems (and data mining) which could have informed the team as to the scope and challenges ahead. As such the local trends and themes could not be determined accurately, a well known issue due to the unquantifiable nature of health visiting practice [8]. NICE [6] guidelines acknowledge the absence of empirical evidence to prove effectiveness of a particular intervention is not the same as evidence for its ineffectiveness [6]. Nonetheless this led to a number of assumptions being made (as to service provision and effectiveness) that needed to be made explicit through the review process.

\section{Listening visits}

Listening visits are a major part of the health visiting ICP to

*Corresponding author: Paul Regan, School of Health, University of Central Lancashire, Brook building, Victoria st, Preston, PR1 2HU, United Kingdom, E-mail: PJRegan@uclan.ac.uk

Received November 04, 2011; Accepted January 08, 2012; Published January 10, 2012

Citation: Regan P (2012) Listening Visits to Mothers' Suffering with Mild to Moderate Peri-Natal Depression; Critical Issues of Clinical Supervision, Training and Health Visiting Capacity to Tackle Health Inequalities. Primary Health Care: Open Access 1:107. doi:10.4172/2167-1079.1000107

Copyright: (c) 2011 Regan P. This is an open-access article distributed under the terms of the Creative Commons Attribution License, which permits unrestricted use, distribution, and reproduction in any medium, provided the original author and source are credited. 
Citation: Regan P (2012) Listening Visits to Mothers' Suffering with Mild to Moderate Peri-Natal Depression; Critical Issues of Clinical Supervision, Training and Health Visiting Capacity to Tackle Health Inequalities. Primary Health Care: Open Access 1:107. doi:10.4172/21671079.1000107

Page 2 of 5

mothers' suffering with mild to moderate OND and can be defined as '...non directive counselling delivered at home (in the form of listening visits...' [6] (p10). Health visitors' application of person centred principles however are unique due to the generic nature of health visitor assessment, the context, experience, training, role and principles (Table 1) [3]. Therefore, the practice development team asked, first; what kind of training health visitors' currently received and secondly, what training may be available as a result of the ICP. The second question will be addressed later in the recommendations section. The answer to the first question was simple, currently there was no training nor had there been for some years. Despite research studies reporting the effectiveness of trained and supervised listening visits the national trend appears to be quite different. There is an assumption when reading the NICE guidelines that research findings are implemented [9-16] but the reality is far different [8]. In September 2010, using the Freedom of Information Act (2000) the Patients Association asked all UK Primary Care Trusts to feedback whether they had implemented NICE [6] guidelines. They asked specifically; what level of training does your PCT provide health visitors' in regards to assessing mental health conditions and in giving psychological support during the antenatal and postnatal period? Only Three Trusts $(n=3)$; Luton PCT, Isle of Wight and St Helens and Knowsley responded (Table 2) [17-19] reinforcing the NICE surveys $25 \%$ estimate of PCT's having a fully developed policy implemented for antenatal and postnatal mental health. As little as three days training was typical, challenging the premise of health visiting training and supervision in listening visits and assessment of mental health conditions.

\section{Health visitors' and NICE guidelines}

Generic health visiting practice is a universal service traditionally offered to all parents and families (Table 1) $[6,20]$. Health visiting involves assessment, support of parents, children and families needs and establishing a working relationship are employed to develop trust, rapport and future timely communication with the emphasis on empowerment [6]. Three levels of interaction between a health visitor and mother overlap the working relationship [10]. Firstly, the public face of their work exemplified in the purpose of the health

\section{- Search for health needs}

- Stimulation of an awareness of health needs

- Influence on policies affecting health

- Facilitation of health enhancing activities

Table 1: The Four health visiting principles (Appleton and Cowley, 2008).

\begin{tabular}{|c|c|}
\hline Luton PCT & $\begin{array}{l}\text { "Regional training has been provided to midwives for } \\
\text { recognition of depression and anxiety. The training programme } \\
\text { has now been developed as on line modules to enable roll out } \\
\text { to other front line professionals... training in use of EPDS" }\end{array}$ \\
\hline $\begin{array}{l}\text { Isle of Wight } \\
\text { PCT }\end{array}$ & $\begin{array}{l}\text { "Midwives trained annually at mandatory study day (only } \\
30 \text { mins). Plans to develop more in depth study day for health } \\
\text { visitors and midwives and community midwives (arranged } \\
\text { for } 21 \text { st January)...e-learning package available via training } \\
\text { tracker" }\end{array}$ \\
\hline $\begin{array}{l}\text { St Helens and } \\
\text { Knowley PCT }\end{array}$ & $\begin{array}{l}\text { "Health visitors have } 3 \text { day training in early recognition } \\
\text { and management of postnatal depression, the training also } \\
\text { incorporates information on other common mental health } \\
\text { problems. They are trained to provide "listening visits" (one to } \\
\text { one support visits in the home)" }\end{array}$ \\
\hline
\end{tabular}

Table 2: Patients Association response to Freedom of Information Act (2000). visiting contact (Table 1), secondly, health visitors' draw on their lay knowledge of 'life events' and familiarity with the situation. Thirdly, health visitors' manage hidden personal feelings that may arise within the interaction. The invisible private knowledge gained in life events has a transforming effect for both the health visitors' and clients with no educational model being able to legitimize such knowledge as a resource for clinical practice [10]. These factors will be discussed later with regards to clinical supervision.

Health visitors' routinely screen mothers' in the postpartum period by assessing maternal mood based on a number of situated observations in clinics, home or other settings [6]. Diagnostic questioning includes asking 'during the past month, have you often been bothered by feeling down, depressed or hopeless...have you often been bothered by having little interest or pleasure in doing things? If yes to either questions a third question should be considered; is this something you feel you need or want help with?' [6]. If there are any yes answers then discussion about risk, depression, treatment options and the services available to a mother followed by the use of the Edinburgh postnatal depression score (EPDS) [6]. The EPDS is a ten item questionnaire requiring a mother to self rate her mood over the past week [21]. If the score is over 13 then it is suggested to be a high likelihood the mother is suffering from a mild to moderate depressive illness [21]. If PND is indicated then an ICP package of care is commenced with six home visits planned called listening visits based on person centred principles $[16,22]$. Health visitors' are then recommended to commence a six week ICP package of care $[6,21]$. NICE guidelines then recommend after consent has been obtained referral to a General Practitioner for anti-depressant therapy, brief cognitive behavioural therapy and interpersonal psychotherapy [6].

\section{Evidence based practice}

A number of systematic reviews found little evidence of systematic approaches to PND detection $[11,23,13]$ with NICE (2011) guidelines recommending the use of diverse psychological treatments in the UK such as brief cognitive therapy, interpersonal psychotherapy and listening visits. Despite favourable comparisons with therapies health visiting research has had limited influence on healthcare policy and more research is needed about the role, training and supervision of health visiting and PND [24]. This would significantly reduce the negative outcomes of prolonged and untreated PND [6].

\section{Discussion}

The evidence based literature makes a number of impact claims based on piloted small scale studies emphasising specialised training for UK health visitors' versus what could be considered untrained (psychotherapeutic) interventions by health visitors' [9,12-16]. Unfortunately, these examples of exemplar practice contrast with the assumption of health visitors' efficacy when receiving little or no training on effective listening visits or other therapy methods. Instead, training focuses on ICP and detection using the EPDS reinforced by NICE stating '...staff have supervision and training, covering mental disorders, assessment methods and referral routes, to allow them to follow the care pathways ... (CG 1.2.2.5).

The discussion whether to train or not train health visitors' is confused by similar health visitors' roles performed by nurses' in other countries and claims of effectiveness [13,21]. Dennis [11] Canadian study reinforced a positive and protective role of intensive home visits by nurses' with at risk mothers' in the first six weeks postpartum [25]. This was not proven to be maintained after 16 weeks when visits 
Citation: Regan P (2012) Listening Visits to Mothers' Suffering with Mild to Moderate Peri-Natal Depression; Critical Issues of Clinical Supervision, Training and Health Visiting Capacity to Tackle Health Inequalities. Primary Health Care: Open Access 1:107. doi:10.4172/21671079.1000107

Page 3 of 5

decreased from weekly to monthly and not maintained at 16 weeks. In contrast Morrell et al. [14,16] studies indicated a continued reduction in depressive symptoms at six months when health visitors' were trained in either person centred therapy or cognitive behavioural methods. A randomised controlled trial (RCT) trained health visitors' $(n=89)$ to clinically assess PND symptoms using EPDS in two groups of psychologically informed interventions $[14,16]$. One group were trained in cognitive behaviour principles and the other in person centred principles for 6 days and 4 half days with supervision offered. Common training aims enhanced acquiring positive regard, empathy and more generic skills of therapy. Person centred training focussed on three principles; actualising tendency and human's tendency to fulfil their potential as they mature, a non-directive attitude and therapeutic conditions of change. Health visitors' trained in cognitive principles focussed on unhelpful patterns of behaviours, perceptions or thoughts. Despite a significant reduction in depressive symptoms in all the women in the intervention clusters $(n=271)$ not one theory was proven to be more effective, called the equivalence paradox. Morrel et al. $[14,16]$ reinforce the importance of health visitors' training and clinical supervision and have made available their RCT training materials.

\section{Presencing}

The principles of person centred therapy; self actualising tendency, non directive and attentive listening and empathy are themes that continue to emerge within supervised, trained health visiting and nurse interventions $[9,12,14-16]$. Depending on maternal need nurses' offering non-directive counselling, weekly supportive interactions, and facilitation or referral to support groups had proven effectiveness [23]. The Registered Nurses' Association of Ontario (RNAO) suggest an interesting existential element to person centred practice by reinforcing nurses' develop reflective insight, non-judgemental attitude and empathy when listening to mothers' experiences of PND [23]. The RNAO (2005) suggest the notion of presencing (being-there) is applicable to person centred principles employed by nurses'. From the work of Martin Heidegger presencing relates (in general) to nearness and overcoming the inter-subjective distance we have with one another [26]. This attunement with a mother's PND experience recommends avoiding we leap-in to solve problems or intellectualising and as a result being in-authentic when in the moment [26]. The presencing of being-there is captured well in the following quote '...the quality of attention so that I maybe with you, alongside you, emphasising with you, and yet not losing myself in confluence with you because the dialogue between us both bridges and preserves our difference..." [27] (Reason, 2004, p219). A difficult concept to grasp without further reflective development through clinical supervision [18,28].

\section{Risk of role duality}

The nature of health visitors' work in ensuring mothers' and baby's health and wellbeing suggests the potential for role duality. The fears of a depressed mother if she discloses child protection issues [15] suggests checking the understanding of health visitors' working knowledge of NICE recommendations and what listening visits entails would seem to be in the interests of best practice to allay mothers' fears. However, this identifies a gap in the literature because listening visits requires different kinds of judgement when the health visitor attempts to '.. develop a trusting relationship...explore the woman's ideas...concerns and expectations and regularly check her understanding of the issues...' [6]. This statement suggests a degree of certainty in what a listening visit entails, despite the variability of experiences in individualised home listening visit and does require reflective awareness of the inherent issues $[6,23](\mathrm{p} 10)$.

\section{Clinical supervision}

NICE recommend a process-and-outcome monitoring, to ensure that treatments are delivered effectively (p123) however reference to (clinical) supervision was only found three times in the guidelines, referring to all primary and secondary care health professionals. In contrast, therapists all receive supervision on their therapeutic work [28]. Despite a proven efficacy in promoting quality care clinical supervision remains largely underdeveloped across UK multidisciplinary teams [29] and in nursing it remains strongly bureaucratic and of concern [30]. In generic health visiting two main strands of clinical supervision are in use; the first on clinical supervision of generic work and the second on safeguarding children in need or at risk. The first model of clinical supervision is accessed voluntarily to ensure a health visitor (individually or within group) can choose a peer (or from a small pool of supervisors) with clinical supervision skills [28]. The supervisee brings to the supervision session their own clinical agenda which has the potential for guided and process driven reflective learning [31]. The issue of potential is significant due to the opportunity to reflect and interpret health visiting practice. A process driven model has as underlying principles of autonomy, empowerment and lifelong learning [28] which appear to be at risk within nursing [30]. What may be in place is what could be called a product orientated model meaning the supervisees presenting issues are inclined to be problem centred in order to find solutions within a short timescale. Therefore, in general supervisees may have exposure to one, the other or a mixture of the two with varying benefits depending on their stage of development [28].

The level of supervision training within nursing and health visiting differs significantly across the UK [29,31], as does the number of health visitors' accessing this model of supervision, due in part to it remaining voluntary in nature and affected by resource implications and managerial processes in practice [30]. Within this context a health visitor maybe unlikely to discuss issues such as presencing, listening visits and EPDS checklist within a narrow viewpoint [23]. A second, mandatory clinical supervision model is dominant in the form of caseload supervision for safeguarding children and children in need. Being expert driven, as a form of consultancy supervision with a specialist safeguarding practitioner it may focus on presenting issues, progress reports, report writing, conference attendance and exposure to an organisational model of quality governance [30]. This cannot replace reflective learning as described in the first process driven model of clinical supervision but of course may be effective for this professional context [28].

\section{Cost effectiveness and widening the scope of practice}

Listening visits offered to mothers' suffering with peri-natal depression conclude 'non-directive counselling (listening home visits) are likely to be a cost-effective treatment preference for women with mild to moderate depression in the postnatal period' [6]. Despite this evidence there is continued pressure on health visiting teams to reduce service costs, provide more care in clinics, manage more chronic safeguarding at risk and in need cases and reducing the frequency of home visiting [3]. Mothers' find listening visits has a significant positive impact on infants' cognitive and emotional development, as well as on the 'well-being of their wider family' (p184) [6]. Therefore, health visiting appears to be not only cost and clinically effective but with a proven ability to impact on all of the family's emotional health $[1,6,32]$. 
Citation: Regan P (2012) Listening Visits to Mothers' Suffering with Mild to Moderate Peri-Natal Depression; Critical Issues of Clinical Supervision, Training and Health Visiting Capacity to Tackle Health Inequalities. Primary Health Care: Open Access 1:107. doi:10.4172/21671079.1000107

Page 4 of 5

There is clear evidence of a need for more intensive, regular postpartum visiting by nurses' [11,25] and benefits of first parent intensive support through generic health visiting [3,33]. International evidence of nurses' effectiveness with PND [13,23] suggests health visiting teams may need to consider moving away from a (hierarchical) model of health visitors' only offering listening visits and instead utilise the wider availability and develop the role for community nurses' working in health visiting teams.

\section{Political influence}

Political interference has had a significant influence on the future of health visiting, and not for the public good [8]. The expansion of commissioning [5] appears to have been instrumental in the professions reduced capacity to tackle health inequalities. This was evident in 2006/07 when PCT's failed to fund enough training (329 in spite of 798 applicants) [33]. As the health visiting workforce reduced, $£ 200$ million was allocated to develop outreach workers from the Children's centres [33], money now implemented to fund more health visitor training [1]. As well as the McKinsey report [4], the threat to remove health visitors' registerable qualification in the year 2000 should have rang alarm bells much sooner. The expansion of commissioning, with international healthcare providers being allowed to tender for NHS services paid for by the UK taxpayer [34] adds to the risk of slow implementation of NICE guidelines and well funded local action [5].

\section{Conclusion}

Listening visits are an effective supportive intervention within health visiting aimed at the early detection of women suffering from per-natal depression, and drive towards cost and clinically effective practice reinforcing its potential as a proven therapeutic intervention [6]. The issue of whether to train health visitors' or not in listening visits appears to be difficult to quantify but PCT's have responsibilities to promote quality governance through evidence based practice [6]. However, NICE guidelines don't go far enough to recommend what amount or level of health visitor training, education and clinical supervision with evidence promoting the role of community nurses' and PND. Therapeutic models such as cognitive behavioural therapy and person centred principles by trained health visitors' are effective but what makes listening visits by health visitors' so unique is the universal, timely and proactive early intervention within the postnatal care $[1,34]$. This last point reinforces a need for a well resourced and commissioned health visiting service, far removed from political interference, and a renewed capacity for the profession to tackle national health inequalities [1].

\section{Recommendations}

- PCT's should develop local protocols and action plans as per NICE guidelines.

- Health visitors' need training in the assessment of mental health, use of ICP's and person centred principles for listening and/ or cognitive behaviour material. Training should be comprehensive and follow Morrell et al. [14,16] model with atleast six full days, four half days and supported development through clinical supervision.

- Health visitors' require a process driven clinical supervision model for PND cases. This model should be in addition to other models, therefore compliment supervision for safeguarding children.
- Based on local need, in relation to PND evidence should be readily available for commissioning of health visiting services.

- Health visiting research has had limited influence on healthcare policy. More research is required to underpin and inform the role of the health visitors' practice.

- The scope for non health visitors' (nurses') working within community teams should be expanded as indicated by the international research findings.

\section{References}

1. Department of Health (2011) The health visitor implementation plan 20112015; a call to action. London: HMSO.

2. Department of Health (2009a). Transforming Community Services: Enabling new patterns of provision. HMSO, London.

3. UKPHA (2009) Health visiting matters; re-establishing health visiting, United Kingdom Public Health Association.

4. Department of Health (2009b) Achieving world class productivity in the NHS 2009/10 - 2013/14: Detailing the size of the opportunity. McKinsey and Co.

5. Regan P, Ball E (2010) Liberating the NHS; commissioning, outsourcing and a new politics debate. Br J Community Nurs 15: 503-505.

6. National Institute for Health and Clinical Excellence (2007) Antenatal and postnatal mental health; the NICE guideline on clinical management and service guidance. London.

7. Sockol LE, Epperson CN, Barber JP (2011) A meta-analysis of treatments for perinatal depression. Clin Psychol Rev 31: 839-849.

8. Regan $P$ (2011) Critical issues in practice development; identifying with localism and the new public health reforms. Community Pract 84: 32-35.

9. Davies BR, Howells S, Jenkins M (2003) Early detection and treatment of postnatal depression in primary care. J Adv Nurs 44: 248-225.

10. Pritchard JE (2005) Strengthening women's health visiting work with women. J Adv Nurs 51: 236-244.

11. Dennis C (2005) Psychosocial and psychological interventions for prevention of postnatal depression: systematic review. BMJ 331: 15.

12. Shakespeare J, Blake F, Garcia J (2006) How do women with postnata depression experience listening visits in primary care? A qualitative interview study. Journal of Reproductive and Infant Psychology 24: 149-162.

13. McQueen K, Montgomery P, Lappan-Gracon S, Evans M, Hunter J (2008) Evidence-Based Recommendations for Depressive Symptoms in Postpartum Women. J Obstet Gynecol Neonatal Nurs 37: 127-136

14. Morrell CJ, Slade P, Warner R, Paley G, Dixon S, et al. (2009) Clinical effectiveness of health visitor training in psychologically informed approaches for depression in postnatal women: pragmatic cluster randomised trial in primary care. BMJ 338: a3045.

15. Turner KM, Chew-Graham C, Folkes L, Sharp D (2010) Women's experiences of health visitor delivered listening visits as a treatment for postnatal depression: a qualitative study. Patient Educ Couns 78: 234-239.

16. Morrell CJ, Ricketts T, Tudor K, Williams C, Curran J, et al. (2011) Training health visitors in cognitive behavioural and person-centred approaches for depression in postnatal women as part of a cluster randomised trial and economic evaluation in primary care: the PoNDER trial. Prim Health Care Res Dev 12: 1-10.

17. Luton Primary Care Trusts (2010) Postnatal Depression.

18. Isle of Wight NHS Primary Care Trust (2010) Postnatal depression.

19. St Helens and Knowley Primary Care Trust (2010) Postnatal depression.

20. Appleton JV, Cowley S (2008) Health visiting assessment principles - unpacking critical attributes in health visitor needs assessment practice: a case study. Int J Nurs Stud 45: 232-245. 
Citation: Regan P (2012) Listening Visits to Mothers' Suffering with Mild to Moderate Peri-Natal Depression; Critical Issues of Clinical Supervision, Training and Health Visiting Capacity to Tackle Health Inequalities. Primary Health Care: Open Access 1:107. doi:10.4172/21671079.1000107

21. Cox JL, Holden JM, Sagovsky R (1987) Detection of postnatal depression; development of the 10 item Edinburgh postnatal depression scale. $\mathrm{Br} \mathrm{J}$ Psychiatry 150: 782-786.

22. Holden JF, Sagovsky R, Cox JL (1989) Counselling in general practice setting; a controlled study of health visitor intervention in treatment of postnatal depression. BMJ 298: 223-226.

23. Registered Nurses Association of Ontario (2005) Interventions for postpartum depression. RNAO. Toronto, Ontario, Canada.

24. Bunn F, Kendall S (2011) Does nursing research impact on policy? A case study of health visiting research and UK health policy. Journal of Research in Nursing 16: 169-191.

25. Armstrong KL, Fraser JA, Dadds MR, Morris J (1999) A randomized, controlled trial of nurse home visiting to vulnerable families with newborns. J Paediatr Child Health 35: 237-244.

26. Boss M (1987) Heidegger; The Zollikon seminars. Northwestern University Press, Evanston, Illinois.

27. Finlay $L$ (2011) Phenomenology for therapists; researching the lived world. Wiley-Blackwell, Oxford.

28. Hawkins $P$, Shohet $R$ (2006) Supervision in the helping professions, $3^{\text {rd }}$ edition, Open University Press, Milton Keynes.

29. Bishop V (2008) Clinical governance and clinical supervision; protecting standards of care. Journal of Research in Nursing 13: 3-5.
30. Yip K (2006) Self-reflection in reflective practice; a note of caution. British Journal of Social Work 36: 777-788.

31. Cutcliffe JR, Hyrkas K, Fowler J (2011) Routledge handbook of clinical supervision; fundamental international themes, Routledge, Oxon.

32. Hay DF, Pawlby S, Sharp D, Asten P, Mills A, et al. (2001) Intellectual problems shown by 11 -year-old children whose mothers had postnatal depression. $J$ Child Psychol Psychiatry 42: 871-889.

33. Conservative Research Department (2010) Helping new families: support in the early years through universal health visiting.

34. Department of Health (2010) Healthy lives, healthy people: our strategy for public health in England. London: HMSO.

35. Milne D, Sheikh Al, Pattison S, Wilkinson A (2011) Evidence-based training for clinical supervisors: a systematic review of 11 controlled studies. The Clinical Supervisor 30: 53-71.

36. O'Hara MW, Swain AM (1996) Rates and risk of postpartum depression - a meta analysis. International Review of Psychiatry 8: 37-54.

37. Rogers C (2004) A therapists view of psychotherapists; on becoming a person. Constable and Robinson, London. 